Cahiers de recherches médiévales

\title{
Tristan et Isolde de Wagner, et sa postérité littéraire
}

\section{Timothée Picard}

\section{OpenEdition}

\section{Journals}

Édition électronique

URL : https://journals.openedition.org/crm/1733

DOI : $10.4000 / \mathrm{crm} .1733$

ISSN : 1955-2424

\section{Éditeur}

Honoré Champion

\section{Édition imprimée}

Date de publication : 15 décembre 2004

Pagination : 55-71

ISSN : 1272-9752

\section{Référence électronique}

Timothée Picard, «Tristan et Isolde de Wagner, et sa postérité littéraire », Cahiers de recherches

médiévales [En ligne], 11 | 2004, mis en ligne le 10 octobre 2007, consulté le 15 décembre 2022. URL http://journals.openedition.org/crm/1733 ; DOI : https://doi.org/10.4000/crm.1733

Ce document a été généré automatiquement le 15 décembre 2022.

Tous droits réservés 


\title{
Tristan et Isolde de Wagner, et sa postérité littéraire
}

\author{
Timothée Picard
}

1 Voici ce que Denis de Rougemont déclare dans L'amour et l'Occident à propos de Tristan et Isolde de Wagner, opéra en trois actes créé au Hoftheater de Munich le 10 juin 1865 : «Que Wagner ait restitué le sens perdu de la légende, dans sa virulence intégrale, ce n'est point là une thèse à faire admettre, c'est l'évidence largement déclarée par la musique et les paroles de l'opéra. Par l'opéra, le mythe connaît son achèvement. [...] Achèvement désigne l'expression totale d'un être, d'un mythe ou d'une œuvre ; d'autre part, désigne leur mort. Ainsi le mythe “achevé” par Wagner a vécu. Vixit Tristan! Et s'ouvre l'ère de ses fantômes " ${ }^{1}$. Comment la littérature européenne a pu voir dans le chef-d'œuvre wagnérien une incarnation stérilisante de l'organon absolu au sens de Schelling, à savoir une œuvre qui « sature l'horizon de l'art tout en l'accomplissant ", et comment elle s'est peu à peu émancipée de ce modèle grâce à la redéfinition de la fable d'Eros et de sa dramaturgie, tel sera notre propos ici.

Les sources médiévales et leur reprise par Wagner

2 Wagner reconnaît lui-même, non sans effroi, la puissance émotionnelle de son œuvre : « Tristan devient quelque chose de terrible ! Ce dernier acte !... J'ai peur qu'on interdise mon opéra, sauf si de mauvaises représentations en donnent une parodie. Elles seules lui assureront la vie sauve. Car si elles étaient parfaitement bonnes, les gens deviendraient fous... $»^{2}$. Pour autant, il ne révèle pas un quelconque souhait de parvenir à la pleine réalisation et donc à l'accomplissement du mythe tristanien. Bien au contraire, même, si l'on en croit le Prologue qu'il rédige pour Tristan et Isolde: «Un ancien poème d'amour originel, se recomposant sans cesse de nouveau sans perdre de son souffle, adapté en toutes les langues de l'Europe médiévale, nous raconte l'histoire de Tristan et Isolde. [...] Le musicien qui choisit ce thème comme ouverture à son drame d'amour, se sentant alors entièrement pris dans l'élément illimité spécifique à la musique, pouvait s'inquiéter uniquement de savoir à quoi il se bornerait à l'intérieur de ce thème qu'il est impossible d'épuiser ${ }^{3}$. Wagner ne se présente lui-même que comme le continuateur d'une matière pluriséculaire. D'où vient alors cette réputation de 
perfection qui, à travers toute l'Europe du XXe siècle, chez ses thuriféraires comme chez ses détracteurs, de Nietzsche et Adorno à Bloch ou Marcuse, de T. Mann, Hesse et Schnitzler à Broch ou Musil, de Claudel, Valéry, Rolland ou Pourtalès à Leiris, Gracq ou Robbe-Grillet, de D'Annunzio à Balestrini, chez Lawrence, Joyce ou Woolf, Blok, Ivanov ou Bielyï, caractérise l'œuvre?

Elle tient tout d'abord à l'épure du livret, sans aucune mesure avec la pratique de l'époque mais également sans relation avec les péripéties diverses qui caractérisent les sources médiévales. Wagner a fréquenté de façon assidue les textes médiévaux relatifs au mythe dès les années 1843-1849, à savoir deux éditions du roman de Gottfried von Straßburg (1205-1210), les continuations de Ulrich von Türheim (1235) et de Heinrich von Freiberg (1280), les chansons d'amour du même Gottfried, Sir Tristem en moyenanglais d'après Thomas von Erceldoune, le Tristan de Béroul (1180), le Lai du chèvrefeuille de Marie de France, un poème gallois et le fragment de Magdebourg du Tristant d'Eilhart von Oberg (1170). Toutefois, il ne forme le véritable projet d'un Tristan qu'en 1854. Une célèbre lettre à Liszt en rend compte : «Comme dans mon existence je n'ai jamais goûté le vrai bonheur que donne l'amour, je veux élever à ce rêve, le plus beau de tous, un monument dans lequel cet amour se satisfera largement d'un bout à l'autre. J'ai ébauché dans ma tête un Tristan et Iseult. C'est la conception musicale la plus simple, mais la plus forte et la plus vivante $»^{5}$. Pour que ce projet prenne forme, et se convertisse en ébauche de livret en 1856, il a fallu plusieurs éléments déclencheurs dont les exégètes de Wagner s'accordent à dire qu'il est difficile d'en mesurer la part respective, et même le rôle véritable : la lecture (inégalement attentive et en tous cas partiale) du Monde comme volonté et comme représentation de Schopenhauer (Die Welt als Wille und Vorstellung, 1818) ${ }^{6}$, la rencontre amoureuse avec l'épouse d'un de ses mécènes, Mathilde Wesendonck, prétexte à une expérimentation des mécanismes de la passion plus que passion véritable, enfin l'émulation-stimulation de l'ami Karl Ritter, qui songeait lui aussi à écrire un drame sur le sujet de Tristan. Les lieux de composition, en particulier Venise pour le deuxième acte, jouèrent un rôle important dans la postérité littéraire et philosophique de l'œuvre?.

Wagner condense passablement les sources originales. L'acte I présente le retour de Tristan vers la Cornouaille, accompagné d'Isolde, et leur ingestion du philtre ; l'acte II, la nuit d'amour interrompue par l'arrivée de Marke, averti par un traître ; et l'acte III, Tristan blessé qui, ayant fui sur ses terres, attend l'arrivée d'Isolde et meurt, bientôt rejoint par sa bien-aimée. Les récits de l'acte I (Isolde à Brangäne) et de l'acte III (Tristan à Kurwenal ou, délirant, à lui-même), permettent d'accéder à la préhistoire du drame, dont ne sont donnés que les aspects essentiels, hissés à une valeur symbolique : mort des parents de Tristan, naissance de l'amour-haine d'Isolde pour Tristan, meurtrier de son fiancé. S'il ajoute les personnages épisodiques du marin (acte I) et du berger (acte III), le compositeur librettiste fait disparaitre le personnage d'Isolde-auxblanches-mains, concentre ceux des « losangiers " (les dénonciateurs) en le seul Melot, fait disparaître le dragon ennemi au profit de l'unique Morolt, fond les différents trajets en bateau. Plus : il donne davantage de poids à certaines figures et défait de tout caractère contingent des actions déterminantes pour le drame. Ainsi, ce Morolt que tue Tristan n'est plus l'oncle mais le fiancé d'Isolde; Marke n'est pas, comme chez Gottfried, une figure assez prosaïque de l'amour sensuel mais un homme prônant le pardon et le renoncement; les philtres d'amour et de mort sont échangés non par maladresse mais par intention délibérée; enfin, l'amour de Tristan et d'Isolde précède 
l'ingestion du philtre. De façon générale, l'action ainsi épurée permet une focalisation du spectateur sur l'entier épanchement des intériorités tourmentées et désirantes. Pour autant, chaque acte étant dynamisé par une attente (craintive ou euphorique : arrivée à la cour de Marke à l'acte I ; irruption de la cour à l'acte II ; attente d'Isolde à l'acte III), l'œuvre conçue comme une arche à trois courbes, jouant sur des effets de dilatation et de compression temporelles mimétiques d'un désir à la recherche d'une résolution toujours repoussée, évite tout statisme. Wagner, dans la rédaction du livret, s'est beaucoup inspiré du vocabulaire, de la syntaxe et de la métrique du moyen-haut allemand; tout le deuxième acte (la nuit d'amour menacée par le jour) reprend le canevas de la chanson d'aube.

5 En plus de ces sources médiévales, qui offrent à Wagner la possibilité de formuler une conception personnelle de l'union mystique propre à la religion chrétienne de la fin du $\mathrm{XII}$ siècle, on note d'autres champs d'influence, pas toujours aisément identifiables parce que toujours réinterprétés et fondus dans un ensemble: Calderón, les spiritualités orientales (bouddhisme et brahmanisme), et, des Hymnes à la nuit de Novalis à la Lucinde de Schlegel et surtout Schopenhauer, le romantisme allemand, philosophique et poétique. Ce syncrétisme spirituel flou mais capable cependant de prendre, enrobé de musique, un caractère d'évidence dans le temps de la représentation théâtrale, à laquelle il donne un halo métaphysique, n'est pas anodin dans la fortune de l'œuvre.

Un sommet du romantisme musical allemand

6 Si le Tristan de Wagner a pu donner le sentiment de l'œuvre parfaite, c'est aussi parce qu'il condense et pousse à leur paroxysme certaines données fondatrices du romantisme musical allemand; ceci, en faisant parfaitement coïncider le fond du drame (une métaphysique d'Eros) et les moyens musicaux et dramatiques permettant d'en rendre compte (le mythe, l'œuvre d'art totale, la mélodie continue, le leitmotiv, le chromatisme et la dissonance). En ce sens, par le biais du lyrisme, point de rencontre entre expression du Moi et art lyrique, Wagner ajuste idéalement les moyens dont il dispose aux fins qu'il s'est prescrites : la revivification du mythe tristanien. La musique en particulier, fluide idéal en ce qu'elle est à la fois concrète et immatérielle, tantôt symbole de la Volonté universelle (Schopenhauer), de l'effet total du dionysiaque dans la tragédie (Nietzsche), des tensions et conciliations entre Eros et Thanatos (Rougemont), etc., est élevée au niveau d'une métaphysique en acte. Métaphysique wagnérienne de la musique et conception wagnérienne du monde en général, fondée sur le pessimisme historique (la dégénérescence inexorable du monde vient des ruses érotiques du Vouloir-vivre), ne font ici qu'une seule et même chose.

Ce que Tristan représente, c'est donc la quintessence de la douleur d'être au monde selon l'homme romantique. Celle-ci se révèle tout d'abord dans l'insuffisance de ce monde, source autant de désir que d'insatisfaction pour un Moi aspirant à l'infini. Le primat de la subjectivité et de l'intériorité, l'inclination vers les puissances obscures de l'irrationnel, la quête de l'idéal (l'Un et le Total), viennent de cette volonté de surmonter le drame de l'individuation et du souhait de se dissoudre dans le grand Tout cosmique. L'opposition symbolique qui structure le drame, entre le jour et la nuit, en est l'expression métaphorique : la nuit est ainsi ce qui permet aux amants d'échapper au monde social, phénoménal et pluriel, et de s'enfoncer en direction du point où, dans l'extase inconsciente de l'unité originelle retrouvée, s'unissent Eros et Thanatos. Si Eros et Thanatos ne s'opposent plus, c'est que l'individu, confronté à l'expression absolue 
d'Eros, découvre que seule la mort est capable de lui donner l'éternité à laquelle Eros aspire. Surmonter le drame de l'individuation, c'est abolir les limitations spatiotemporelles du sujet, et rendre à la fois possibles l'exercice de la subjectivité et l'accession à la transcendance. En ce sens, seule la musique, art du temps mais capable de transcender le temps, est en mesure de donner l'image de l'unité rédemptrice et salvatrice. Elle donne au mot un caractère incantatoire par lequel le texte se résout dans la musique, en une fusion qui est justement l'image même d'une conciliation entre l'orgiasme anarchique d'Eros et sa figuration plastique rassérénée. Conjointement, c'est aussi l'image d'une tension et d'une résolution entre les différents pôles de l'érotique wagnérienne : le sensualisme feuerbachien, le pessimisme schopenhauerien, l'agapè et le renoncement d'un christianisme sur lequel ont soufflé certains vents d'Orient. À ceci près qu'ici les souffrances désirantes des protagonistes montrent assez, à la façon presque d'une moralité, le triomphe d'Eros et ses ravages. Incapables de déchirer le voile de Maïa, l'illusion universelle, Tristan et Isolde ne sont que les incarnations conjoncturelles du grand désir ressassé, d'une Volonté primordiale, qu'ils aiment davantage qu'ils ne s'aiment eux-mêmes.

À en croire le T. Mann des Considérations d'un apolitique (Die Betrachtungen eines Unpolitischen, 1918) ou de La montagne magique (Der Zauberberg, 1924), il faudrait ajouter que cette métaphysique romantique aurait une spécificité germanique qui se révélerait dans cette "affinité avec la mort » dont fait preuve Tristan, une affinité seule capable d'opérer cette " poétisation de l'éthique " qu'il considère comme tellement étrangère à l'esthétisme méditerranéen. De fait, en plus des influences de Novalis, Schlegel ou Schopenhauer, déjà citées, la poétique wagnérienne semble synthétiser les points fondamentaux de plusieurs grandes pensées du romantisme allemand. Grâce à Schlegel, Wagner comprend la nécessité de ressusciter la mythologie, voire de créer une nouvelle mythologie. Grâce à Hegel et Schelling, il prend conscience que le "grand art " romantique doit accomplir la promesse esthético-politique de l'art, telle qu'héritée des Grecs. De la pensée de Herder, il tire l'idée que le mythe, particulièrement sous sa forme musicale, est l'émanation poétique la plus authentique du peuple. Feuerbach et Bakounine apportent à ces influences une dimension pédagogique et révolutionnaire. Enfin, avec Fichte, il fait sienne l'idée d'un messianisme européen de l'art et de la culture allemands.

Théorisations de « l'amour en Occident » à partir de Wagner

La question de la "germanité » de la matière tristanienne a en effet son importance au tournant $\mathrm{du} \mathrm{XIX}^{\mathrm{e}}$ et $\mathrm{du} \mathrm{XX}^{\mathrm{e}}$ siècle, époque où il est particulièrement exigé du mythe qu'il donnât à un peuple, à l'exclusion des autres, sa forme esthético-politique parfaite, spirituellement valable sur le plan européen. Ainsi, de Rossetti à Swinburne et Yeats en Grande-Bretagne, de Nietzsche à T. Mann en Allemagne, de Mendès et Péladan à Debussy, Rolland, Suarès et Giraudoux en France, chez Pourtalès en Suisse, on revendique, pour sa patrie, ou pour le modèle culturel auquel on prétend appartenir, la paternité du mythe. On se prend à rêver d'un Tristan anti-wagnérien, anti-germanique ; on accuse Wagner d'avoir germanisé, vampirisé et perverti une matière qui serait typiquement française ou latine; on souhaite également l'écriture d'un Tristan shakespearien.

Plus largement, le Tristan de Wagner donne lieu, dès la fin du XIX ${ }^{e}$ siècle et tout au long du siècle suivant, à des interprétations majeures, visant à (re)définir, pour le devenir européen, l'essence du théâtre, la nature et la fonction d'Eros, celles du mythe, et ce, 
sous le triple angle métaphysique, historique et social. Les quatre textes wagnériens de Nietzsche concèdent une place importante à Tristan. Dans la Quatrième considération inactuelle (Unzeitgemässe Betrachtungen, 1876), l'œuvre est qualifiée de "véritable opus metaphysicum de tout art ", parce qu'à travers elle peut se lire la renaissance de ce «dramaturge dithyrambique » que le socratisme de civilisation avait, depuis l'antiquité grecque, réduit à néant et qui s'avère nécessaire pour que «l'état d'âme tragique ne périsse pas ». C'était déjà le sens de La naissance de la tragédie (Die Geburt der Tragödie, 1872), texte dans lequel le philosophe rend compte, en s'aidant de l'exemple wagnérien, du «but suprême » de la tragédie, comme de l'art en général : "permettre que chaque individu, grâce au glacis plastique apporté in extremis par l'élément apollinien (le mythe imageant), ait accès au déchaînement orgiaque de l'élément dionysiaque (la musique en tant qu'universelle expression de la souffrance du monde)», sans que, dans cet " immense mouvement symphonique », "il ne suffoque sous la tension convulsive de toutes les ailes de l'âme ». Ainsi, grâce à ce processus tragique, il serait mené jusqu'à cette "limite où le monde phénoménal se nie lui-même " et "donne à pressentir derrière lui, à travers son anéantissement, une joie esthétique plus haute - une joie originelle au sein même de l'Un originaire ». Tel, alors, serait le sens de ce "chant du cygne métaphysique » qu'est la mort d'Isolde: la tension et pourtant «l'alliance fraternelle des deux divinités », la dissolution du mythe apollinien dans « la formidable pulsion » de " la sagesse dionysiaque ", afin qu'un art authentique ait enfin à nouveau lieu. Le cas Wagner (Der Fall Wagner, 1888) et Nietzsche contre Wagner (Nietzsche contra Wagner, 1889) signent l'émancipation du philosophe par rapport à la tutelle wagnérienne. Tristan, moins concerné par la question chrétienne du salut que le reste de la production wagnérienne, semble relativement épargné par ses sarcasmes, au détriment de Parsifal. Pourtant, c'est essentiellement à Tristan que la littérature postwagnérienne accordera les traits suivants, points récurrents de la critique nietzschéenne appliquée à Wagner et au wagnérisme : maladie, décadence, hystérie, névrose, histrionisme, bovarysme élevé au niveau du mythe, penchant idéaliste pour "l'ineffable", goût pour l'élémentaire et en particulier pour une musique " océanique » dans laquelle le pied léger et dansant de Zarathoustra risque de se noyer etc. T. Mann, dans ses différents essais wagnériens regroupés rétrospectivement dans le recueil Wagner et notre temps (Wagner und unsere Zeit), reprendra, amplifiera et adaptera aux exigences du jour les louanges et les critiques de Nietzsche à l'égard du Tristan de Wagner'. Si, comme on l'a dit, la germanité de l'œuvre sera évoquée dans La montagne magique, la question du dilettantisme et décadentisme tristaniens sera glosée dans les nouvelles Tonio Kröger et Tristan (1903), cependant que Le Docteur Faustus (1947) remettra en question les travers du romantisme musical, à la métaphysique trouble, dangereusement sensuelle et possiblement criminelle. L'analyse nietzschéenne du Tristan de Wagner comme exemple de renaissance de la tragédie aura également une influence déterminante sur la pensée européenne du théâtre symboliste, qu'il s'agisse de Dujardin, Schuré, Mendès, Péladan ou Claudel en France, Yeats en Irlande, D’Annunzio en Italie, Blok, Bielyï, Ivanov ou Tchoulkov en Russie, enfin Strindberg en Suède. Ces pensées envisagent la fonction métaphysique, religieuse et politique du théâtre, notamment la possibilité de trouver un équivalent moderne aux antiques Dionysies, et s'intéressent dans ce cadre à la place théorique et pratique de la musique.

Si l'éloignement de Nietzsche par rapport à l'œuvre wagnérienne s'effectue principalement en raison de son christianisme diffus, fruit inévitable d'un romantisme 
qui étouffe « à force de ruminer des absurdités morales et religieuses » c'est, en miroir inverse, en raison d'un christianisme trouble que Claudel, Eliot ou Broch s'en prennent à Wagner, et particulièrement à la mystique amoureuse de son Tristan. Claudel ${ }^{10} \mathrm{a}$ plusieurs fois avoué sa passion de jeunesse pour Tristan: "J'aimais plus que tout Tristan: oh! j'aimais tellement Tristan avec sa nuit et son râle interminable d'agonie sans espoir " ". Mais, bientôt il s'attaque particulièrement à ce qu'il considère désormais comme un "rut public ", et préfère, à la place du drame de la chair conspirant contre l'esprit, trop galvaudé, le drame moins rebattu de «l'esprit qui désire contre la chair », dans toute l'« atroce intensité " ${ }^{13}$ qui est celle de Partage de midi et du Soulier de satin. Dans sa lettre au Figaro intitulée "Le théatre catholique", il explique la carence intrinsèque, métaphysique de Tristan : «La révolte, l'amour humain, la joie de vivre, etc., tout cela compose mal, car ces sentiments sont impuissants par eux-mêmes à agréger la totalité de l'Univers, et provoquent des harmoniques irréductibles. C'est ainsi que le dénouement de Tristan et Isolde m'a toujours semblé mesquin et presque ridicule, et malgré tous les prestiges de la musique, je reste là-devant comme un homme réfractaire au narcotique. L'art purement laïc qui existe depuis la Renaissance a eu son temps, et l'on peut estimer qu'il a épuisé ses résultats " ${ }^{14}$. Chez Eliot, l'inscription des premiers mots de l'opéra de Wagner au début de La terre vaine (The waste Land, 1922) a pour but d'exprimer la laïcisation néfaste d'Eros, devenue pur débordement sensuel, aussi peu fécond pour la spiritualité européenne que la trop abondante reverdie et cette «mort par noyade » qui l'accompagnent inévitablement. Pour Broch ${ }^{15}$, un opéra comme Tristan et Isolde trahit quelque chose de beaucoup plus grave : l'intrusion, par le biais du romantisme, du kitsch en art, système clos du vide des valeurs qui porte rien moins que le visage de l'Antéchrist, non pas ce destructeur d'idoles souhaité par Nietzsche, mais bien celui, apocalyptique, d'Hitler. Parce que l'art est incapable d'assumer sans faille la tension cosmique que le romantisme assigne à l'art depuis que l'homme s'est placé au centre de la création, parce que la bourgeoisie du XIX ${ }^{\mathrm{e}}$ siècle s'est mise, en raison de sa morale ascétique, à mystifier la passion érotique, alors son besoin kitsch de décor s'est exprimé de la façon la plus grave, la plus profonde et la plus cosmique qui soit : le Tristan de Wagner. Ce qui s'est mis à primer est non l'éthique mais la religion de la beauté. La lecture que Denis de Rougemont fait de l'œuvre de Wagner est également intégrée dans une plus large perspective chrétienne, le nécessaire parcours d'Eros à agapè, mais de façon beaucoup moins critique que ne sont celles de Claudel, Eliot ou Broch. Peu importe ici de savoir que l'auteur se réfère à un catharisme dont les médiévistes ont montré depuis ce qu'il avait d'improbable : pour Rougemont, Wagner, plus encore que les auteurs médiévaux qui ont sacrifié à l'épique et au pittoresque, a donné pleinement et définitivement au mythe de Tristan sa parfaite expression. Loin de glorifier le sexuel, ni même de se présenter comme un drame de la sensualité, Wagner, par la musique, a exprimé la brûlure abstraite, mystique, orientale et finalement hérétique, d'Eros. Au refus érotique du monde s'oppose selon l'auteur la vraie passion occidentale : l'amour-action, l'agapè chrétien, dont le mariage est l'un des visages. Il n'empêche: la profanation du mythe après Wagner, sa vulgarisation, constitueraient d'après Rougemont l'une des explications de la crise moderne du mariage - et, surtout, de certains des déferlements mortifères passionnés qui ont caractérisé le $\mathrm{XX}^{\mathrm{e}}$ siècle.

12 Face à ces relectures chrétiennes du Tristan wagnérien se dressent les lectures marxisantes de Brecht et de Marcuse. Pour Brecht ${ }^{16}$, Tristan, œuvre que par ailleurs il 
admirait beaucoup, est par excellence de celles qui utilisent en vue de leur propre glorification le phénomène d'hypnose musicale. Le besoin bourgeois de côtoyer dans l'art le sublime et l'ineffable se dégrade ici en pure marchandisation de la jouissance musicale. La condition historique et sociale du spectateur s'abolit dans l'extase du musicalisme. Grandeur et décadence de la ville de Mahagonny (Aufstieg und Fall der Stadt Mahagonny, 1930), œuvre dans laquelle les mécanismes érotiques qui fondent la société sont exhibés outrageusement, abonde jusqu'à l'écœurement dans le sens de la jouissance culinaire. L'interprétation de Marcuse part de son côté du même postulat que Rougemont - Tristan n'est pas une apologie de l'amour-passion - pour aboutir à la même conclusion que celle de Brecht : en dépit de son apparente permissivité érotique, l'œuvre wagnérienne ne fait au fond qu'abonder dans le sens de la répression bourgeoise des besoins instinctuels, et cela prétendument au nom de la civilisation. Ainsi, parce qu'il "exige le sacrifice du bonheur terrestre " et que «le principium individuationis est présenté comme une faute », « le Liebestod le plus orgastique célèbre encore la renonciation la plus orgastique $»^{17}$. S'attacher au Tristan de Wagner, c'est, à l'intersection entre le politique, le religieux, le métaphysique et l'esthétique, envisager la place d'Eros en Occident, précisément à l'heure où chacune de ces sphères connaît d'importants bouleversements de contenu et de prérogatives.

La représentation de l'Eros wagnérien en littérature

13 En raison de cette théorisation européenne d'Eros à travers Tristan, la représentation que la littérature fait de l'Eros wagnérien est elle-même chargée de significations métaphysiques, politiques, sociales. Son ambiguïté tient à sa capacité à se trouver à la fois du côté de l'interdit et du côté de la transgression. Un premier mode de représentation, illustré de façon diverse par Péladan, Panizza, Louÿs, Beardsley, Adrien Bertrand, Laforgue, voire Joyce, Leiris ou Robbe-Grillet, relève de la polissonnerie licencieuse et démystificatrice. Laforgue compare Tristan à un long coït, Panizza (Tristan und Isolde in Paris, 1900) narre le voyage licencieux de Tristan et Isolde à Paris, ce dernier et Péladan confient leurs expériences wagnéro-masturbatoires pendant l'écoute de l'opéra, les héros de Péladan calment l'excitation érotique que la musique leur procure dans les bosquets de Bayreuth (La victoire du mari, 1889). Ces textes, pour la plupart, visent à dégonfler l'enrobage métaphysique grandiloquent, le sérieux du lyrisme cosmique dont est affublé l'Eros wagnérien. Rendre Wagner grivois, c'est vouloir le défaire de sa prétention à la dramatisation d'Eros. Dans Le cas Wagner, Nietzsche critique de son côté le bovarysme de l'œuvre : Tristan ne raconterait selon lui rien d'autre que l'inclination fatale des femmes mariées pour les chevaliers salvateurs. Wedekind (Le chanteur d'opéra [der Kammersänger, 1909]) met en scène le sex-appeal parfaitement artificiel que l'interprétation de Tristan confère à un ténor wagnérien, par ailleurs d'un cynisme outrancier. Joyce (Finnegans Wake, 1939, notamment au chapitre 12) n'hésite pas à recourir au scatologique et au trivial pour donner davantage de chair à un mythe qui s'est perdu à force d'abstraction. Dans ces différents textes, l'ambition carnavalesque dévolue à Eros est relativement réduite : il s'agit tout autant de choquer, ici, que d'émoustiller.

Plusieurs reprises, surtout quand le stimulant érotique se teinte de religiosité, visent davantage un but transgressif. Certains textes prétendant alors dévoiler le vrai visage de Wagner, un visage fondamentalement pervers, qui entrerait en complète contradiction avec l'austère philosophie d'Eros qu'on lui attribue parfois. L'Eros wagnérien est alors confronté au tabou, à l'interdit. Non contents de s'attacher à ce 
mythe par nature morbide qu'est le mythe de Tristan, dans lequel on aime autant la mort que l'amour, Batilliat et T. Mann, dans Chair mystique (1897) ${ }^{18}$ et Tristan (1903), font effet de redondance : leur Isolde est malade, en un sens non pas symbolique mais réel, et cette maladie devient un déclencheur, un stimulant, voire l'objet même d'amour. Lemonnier (Le succube, 1898) ne rend jouissive la musique wagnérienne que dans la mesure où elle est rehaussée par le fantasme d'une union avec un succube, union qui tarit en un même ravissement décadent les deux flux essentiels : le sang et le sperme. Chez Huysmans, Swinburne, Gracq, Leiris ou Robbe-Grillet, le liquide mystique est d'autant plus désirable que s'y mêlent les larmes et le sang des crimes et des viols, le sexe wagnérien est d'autant plus excitant qu'il est mêlé à l'effroi. Wagner devient l'occasion d'une provocation, d'une transgression toute calculée à l'égard de l'ordre moral, de la société, qui manifeste une révulsion pleine et entière, ou au contraire une fausse pudibonderie, signe finalement de sa propre hypocrisie.

C'est que l'utilisation de l'Eros wagnérien peut servir à une véritable revendication militante en vue de la libération d'Eros, que celle-ci signifiât la haine anarchisante de l'ordre et de la stabilité sociale, ou bien la volonté de défaire la société de ses carcans afin qu'elle ne s'atrophiât pas de ses propres scléroses. Chez Forster, la progressive émancipation de la jeune héroïne de Chambre avec vue (A room with a view, 1908) est liée à sa connaissance et compréhension de Wagner. Chez Wedekind (Musique [Musik, 1906]), le fantasme wagnérien est au contraire la marque d'une condition féminine encore enchainée dans les carcans sociaux, et la critique de Wagner devient le biais incongru d'un vibrant plaidoyer pour l'émancipation de la femme. Chez Panizza, les réécritures érotiques de l'œuvre wagnérienne sont à prendre comme de véritables combats pour la libéralisation des mœurs. En des propos assez semblables, quelques décennies plus tôt, Shaw (Le parfait wagnérien [The perfect wagnerite, 1898]) avait mis en avant la contiguïté qui existe entre les cycles wagnériens de l'or et ceux d'Eros, caractéristiques de la société capitaliste. De façon tout à fait autre, les courants ésotériques du tournant du siècle proposent, en s'inspirant de l'Eros wagnérien, de nouveaux modèles de société, visant également, parfois de façon délirante, une émancipation de la psyché, une nouvelle relation au monde de l'être pulsionnel. C'est le cas chez Péladan, mais aussi chez les anthroposophes de Steiner, ou encore dans les courants jungiens de la psychanalyse. Lawrence (Femmes amoureuses [Women in Love, 1916]), inspiré d'une mythologie wagnérienne qu'il refond dans un plus vaste creuset de références mythologico-sexuelles, propose de nouvelles images de l'être sexualisé, contre les rigidités des catégories traditionnelles de la sexualité.

Si l'on cherche, à travers Wagner, à émanciper Eros, l'Eros wagnérien reste cependant d'abord et avant tout, sous sa forme tristanienne, un Eros douloureux. Cette représentation, infléchie par la figure nietzschéenne de Dionysos, est présente, sous une forme dégradée, dans un certain nombre de romans du tournant du siècle, dont le Triomphe de la mort (Trionfo della morte, 1894) ou Le feu (Il fuoco, 1900) de D'Annunzio, La mort de Siegmund (The Trespasser, 1910) de Lawrence, Fortune (Chance, 1913) de Conrad. On en retrouve également l'expression, teintée de bouddhisme wagnérisant, dans plusieurs œuvres de Hesse. Ses héros, Klein, Klingsor, Knecht, mais aussi Siddharta, hésitent entre l'acquiescement aux forces d'Eros, parfois jusqu'à la mort, et la volonté de surmonter par l'ascèse l'appel d'Eros ; la mort de ces personnages reprend parfois le canevas du Liebestod tristanien. Le visage d'Eros douloureux se trouve également au centre du théâtre de Claudel consacré au couple amoureux : le tourment de la chair, 
tout comme le péché, y deviennent des moyens paradoxaux et détournés pour que s'exprime la Grâce. Dans ces œuvres, Eros et Thanatos ne font plus qu'une seule et même chose. Eros est souffrance; ce qui empêche Eros est également source de souffrance; Eros trouve son accomplissement dans l'éloge de la mort, devenue délivrance.

La question de l'Eros wagnérien est en outre directement liée au religieux. De nombreux drames, romans et poèmes reprendront, de Blok, Bielyï, Schuré, Péladan à D’Annunzio, Valéry, Cocteau ou Gracq, les thématiques chrétiennes de la pensée wagnérienne, qui mettent en relation les manifestations anarchiques du désir et leur conversion en pitié. Ces œuvres peuvent aller dans le sens de la foi dogmatique, avancer des images alternatives à ce dogme (celles d'un christianisme enrichi d'apports spirituels autres), ou enfin refuser la christianisation d'Eros et le christianisme en général. Il peut y être question d'opposer Eros et le Christ, ou au contraire de les réconcilier, de statuer sur la place du sensuel, du charnel, et de l'érotique dans le temps, voire dans la dramaturgie du religieux. De façon plus générale, non plus exclusivement chrétienne, l'Eros wagnérien est mis en relation avec le fait religieux luimême, le besoin de la collectivité d'accéder à sa propre sacralité, à une époque où les cultes traditionnels ont perdu de leur signification. Les cultes alternatifs, puisant à des mouvements spirituels anciens ou étrangers, accordent alors toujours une place déterminante au besoin individuel et collectif de jouissance, de possession, voire de transe. Mais si Eros est célébré, il est aussi condamné. Sa puissance fait peur. On ne compte pas, en effet, les textes post-wagnériens révélant le souhait d'éradiquer le sexuel ou, à défaut, de l'endiguer, que cela soit sous la forme d'une tentation de l'ascèse, ou celle d'un platonisme exacerbé. Le meilleur exemple de cette propension reste le texte très wagnérien de Weininger intitulé Sexe et caractère (Geschlecht und Charakter, 1903).

Liée à cette représentation littéraire contrastée de l'Eros wagnérien, on trouve la question plus spécifique de la jouissance lyrique wagnérienne dont les psychanalystes ont montré qu'elle trouvait son entière expression dans Tristan. Elle est manifeste dans de multiples témoignages, aussi bien chez Baudelaire, Péladan, Louÿs, Mendès, Claudel, Valéry, Gracq ou Quignard, D’Annunzio, Lawrence, Woolf, Conrad ou Joyce, T. Mann, Hesse, Wedekind, Werfel, Brecht ou Broch. Cette jouissance lyrique wagnérienne crée un manque dans l'écriture, auquel seule la référence au champ lexical de la sexualité peut suppléer. Cette jouissance est tantôt associée à l'expression nietzschéenne du sentiment tragique de la vie, tantôt à l'extase religieuse, tantôt aux drogues et à l'érotomanie. Proust a parfaitement montré en quoi la jouissance wagnérienne relevait de la révélation religieuse mais pouvait aussi se mêler à l'expression de la souffrance jalouse ${ }^{19}$. Valéry fonde une importante partie de sa théorie musicale du " Moi écrivant » sur l'expérience de la jouissance wagnérienne. Brecht, enfin, définit le théâtre épique et l'effet de distanciation contre la méthode wagnérienne de la fusion des arts, propédeutique à la jouissance aliénatrice. L'histoire de la jouissance wagnérienne est celle d'un apprentissage complexe : celui des prestiges d'une musique qui, plus que tout autre, en remontre par sa puissance et son efficacité, à une littérature qui tente d'en comprendre les mécanismes afin de les faire siens.

L'esthétisation de la mort : Tristan dans le roman post-wagnérien

19 Il a pu être reproché à Tristan une forme d'exaltation de la mort, qualifiée de façon assez floue de «romantique ». Cette affinité avec la mort, symptôme et agent d'une 
décadence, a pu même être considérée comme un paradigme de civilisation. Elle rencontre non seulement le goût résurgent, à la fin du siècle, pour les philosophies et spiritualités nihilistes ou hyper-idéalistes, mais aussi une véritable affinité artistique et intellectuelle avec la mort initiatrice, venue délivrer le sens de la vie et de l'art. On recense, chez les auteurs wagnériens, beaucoup d'œuvres au climat funèbre et mettant en scène des candidats au suicide. Pour ne citer qu'eux: Le feu de D'Annunzio; Les Buddenbrook, Tristan, La mort à Venise (Der Tod in Venedig, 1913), La montagne magique de T. Mann; Le loup des steppes (Der Steppenwolf, 1927), Siddharta, Le jeu des perles de verre (Das Glasperlenspiel, 1943) de Hesse ; La traversée des apparences (The voyage out, 1913), Mrs Dalloway, Les vagues (The waves, 1931) de V. Woolf ; Un beau ténébreux, Le rivage des Syrtes, Un balcon en forêt de Gracq. Toutes ces œuvres mettent en avant les thématiques du crépusculaire, du corps souffrant, de l'aquatique, du nocturne, en un style que l'on peut essentiellement qualifier de musical tendant au lyrique.

Trois œuvres fort semblables permettent particulièrement de se rendre compte de ce tristanisme morbide: Le triomphe de la mort de D'Annunzio, La mort de Siegmund de Lawrence, et Klein et Wagner (Klein und Wagner, 1919) de Hesse ${ }^{20}$. Ces trois œuvres s'achèvent par le suicide du personnage principal, fatalité inexorable préparée par de nombreuses pierres d'attente. Les thèmes de la fuite, sous la forme du retrait ou de l'exil, et du désir, sont omniprésents et entrelacés. Ces œuvres sont pétries de wagnérisme, qui sert non seulement de révélateur, de déclencheur du geste suicidaire, mais dont le style lyrique vient également sublimer ce dernier, l'élever à une dimension presque épique. Ainsi, par exemple, de la mort de Klein, décalquée sur le Liebestod d'Isolde. La musique wagnérienne invite à plusieurs commentaires sur la musique cosmique, dont l'harmonie ou la dissonance, la rythmie ou la dysrythmie, se répercutent sur les corps. Le monde est pensé comme un vaste cœur qui fait battre alternativement pulsion de vie et de mort. L'eau est partout présente, liquide mystique ou maternel, décrit de façon plus abstraite que concrète, lié au philtre tristanien, et dans lequel on se purifie, se réfugie, se perd. L'eau musicale, panthéiste, constitue, avec ses mouvements de tension et de détente, la métaphore du désir. Sa substance mouvante, impalpable, est celle de l'indécidable, mais aussi de la contradiction, de la cyclothymie. Dans tous les cas, références wagnériennes et considérations sur la nature vont de concert : tel environnement aquatique et brumeux, telle forme de rocher, tel clair de lune, tel son de la nature, appellent immédiatement la référence à Wagner. Le tristanisme est une esthétisation mythico-cultivée du réel. De même, le thème de l'attirance pour la mort, jusqu'au saut fatal, scande en permanence le texte, la plupart du temps souligné avec une forme de ressassement complaisant. On aime à se représenter l'éventualité de sa propre mort, le rite funèbre auquel elle donnera lieu.

Dans ces œuvres, où règnent emphase, lyrisme, phrases à la périodisation musicale et océanique, termes relevant d'une métaphysique à la substance floue mais rehaussée jusqu'au symbole par le prestige d'une majuscule qui les poétise et les sacralise, la littérature a aligné sa rythmique et ses harmoniques sur ceux du grand opus wagnérien et fait sienne cette fascinante dangerosité dans laquelle se perd l'être hypnotisé et euphorique. L'œuvre wagnérienne, déclarée dangereuse car demandée par une catégorie d'êtres faibles dont elle signe moralement puis physiquement l'arrêt de mort, a rendu également dangereuse toute une littérature qui en amplifie les effets. Si le tristanisme est une maladie qui représente un danger de civilisation, alors il faut un antidote. Cet antidote, avant d'être celui d'un appel à une nouvelle forme de musique, 
c'est la littérature qui sera en charge de le créer. D'amplificateur des mirages métaphysiques morbides de l'œuvre wagnérienne, menacé par le risque de la boursouflure et $\mathrm{du}$ ridicule, l'écrit devient son contempteur. Sortie de l'eau, la littérature se fait plus incisive : c'est le début du roman de l'énergie, versant ensoleillé du roman wagnérien décadent. Dépasser le tristanisme devient alors une invite à dépasser le romantisme comme posture humaine transhistorique.

La représentation du couple amoureux tristanien dans le théâtre post-wagnérien

Le Tristan de Wagner est particulièrement lié à la question de l'expression du lyrisme dans l'Europe post-romantique. La thématique de l'« unfullfiled love » dans l'Angleterre préraphaélite puis dans la Barcelone moderniste, de la «Bella Donna» dans le symbolisme russe, le renouveau du celtisme en France et en Irlande, ou de la mystique du couple amoureux en Italie, n'auraient pas eu une telle importance s'il n'y avait eu le Tristan de Wagner. Rappelons d'ailleurs ici que les réécritures tristaniennes effectuées par Bédier trahissent elles-mêmes d'innombrables traits de plume wagnérisants ${ }^{21}$. Tristan a intimé, en plus d'un réinvestissement des mythes médiévaux et d'un intérêt pour la philosophie de l'amour courtois, la recherche d'une nouvelle langue poéticomusicale qui soit à nouveau capable de célébrer la mystique de l'amour. Il est évidemment un lieu, au confluant entre poésie et théâtre, où la question du lyrisme posée par le Tristan de Wagner est particulièrement importante : c'est celui de l'opéra. Tristan, à l'opéra, pose moins le défi de la recherche d'une nouvelle philosophie d'Eros, même si celle-ci peut être sensible dans certaines œuvres, que celui du strict duo d'amour comme passage obligé dont il faut désormais redéfinir les formes et l'écriture. Avec la dimension et l'enjeu métaphysique exceptionnels du duo d'amour wagnérien, les compositeurs européens veulent repenser cette scène déterminante, et rivaliser avec elle. Cela, jusqu'à l'excès (excès d'Eros, excès de lyrisme), puis jusqu'au rejet (refus ou réécriture du duo d'amour).

Il existe par ailleurs un certain nombre de pièces de théâtre directement placées sous le patronage du Tristan de Wagner. Parmi celles-ci, on peut particulièrement citer Axël de Villiers de l'Isle-Adam (1872-1889), la trilogie Antonia de Dujardin (1899), Les Ombres sur la mer (The Shadowy waters, 1911) de Yeats, Parisina de D'Annunzio (1913), Pelléas et Mélisande de Maeterlinck (1892), La rose et la croix de Blok (1913) et Partage de midi de Claudel (1906) ${ }^{22}$. Ces différentes pièces ont ceci de commun que, à la suite de Wagner, en relation directe avec lui, elles s'attachent, dans un cadre médiévalisant et à partir d'un nouveau type de relation entre texte et musique, à représenter une certaine mystique, une certaine métaphysique du couple amoureux. Le patronage tristanien, sinon wagnérien, est souvent mis en abyme dans les pièces elles-mêmes par l'intermédiaire d'un objet: le «livre d'amour», parfois désigné explicitement comme «Roman de Tristan ». En-dehors de ce premier champ de ressemblances, on peut établir cinq autres types de ressemblances directes, témoignant de l'influence déterminante du Tristan de Wagner.

La première ressemblance est actancielle. Les pièces en question mettent en scène un couple illégitime, rapidement perceptible comme tel, saisi comme malgré lui par un amour exalté et que l'interdit, le plus souvent incarné par la présence de l'époux légitime, exacerbe, ce qui amène à faire de la glose de cet amour et des possibilités de sa réalisation l'enjeu déterminant de la dramaturgie. La deuxième ressemblance relève de l'onomastique. Un lien sonore s'établit sans conteste de Isolde (Iseult) à Mélisande, Parisina, Ysé, Isaure ou Yzel (dans Le fils des étoiles, « wagnérie kaldéenne » de Péladan 
datant de 1892); de Morold-Melot à Gaulod; de Marke à Arkel, Archambault, ou Amalric. Il circule également entre les couples amoureux : Tristan et Isolde, Pelléas et Mélisande, Ysé et M esa, mais aussi, parfois, comme pour fausser l'attente, entre l'héroïne et l'époux légitime : Ysé et de Ciz. Cette homogénéité onomastique a plusieurs finalités : souligner l'influence wagnérienne, ou à défaut le recours à un même référent médiévalisant ; créer ainsi une musicalité des noms, magique, intemporelle, qui éloigne le couple de toute forme de réalisme. La troisième forme de ressemblance est thématique. Toutes ces œuvres mettent en avant des motifs présents et déterminants dans l'œuvre wagnérienne: la liquidité, le trajet marin, le philtre, la blessure, la chevelure féminine, la dialectique entre le jour et la nuit, la dialectique entre la joie et la souffrance, etc. Le quatrième type de ressemblances relève de la dramaturgie. Parmi les éléments saillants, rappelons essentiellement : le trajet vers l'époux d'une femme et d'un homme qui ne s'aiment apparemment pas et boivent l'équivalent d'un philtre de mort (d'amour) ; la scène pendant laquelle, près de la torche qui va servir de signal aux amants et tandis que les échos de la chasse (ou un substitut) retentissent au loin, une confidente met en garde sa maîtresse inattentive ; le duo d'amour exalté, avec son éloge de la nuit et son dégoût du monde ; la dénonciation du couple clandestin ; la blessure de l'amant attendant son aimée et divaguant ; la mort d'amour. Si ces données ne sont pas intégralement présentes dans les pièces en question, elles sont suffisamment nombreuses pour que le patronage tristanien ne fasse pas de doute. Le dernier type de ressemblances est évidemment représenté par le lyrisme des œuvres en question.

En dépit de ressemblances évidentes, ces pièces tristaniennes sont malgré tout en dissidence par rapport au modèle wagnérien. Dépasser Wagner veut avant tout dire dépasser Tristan. Cela peut tout d'abord se traduire par le refus de la dimension tout uniment mythique et sublime du couple tristanien, et la réintroduction de certains éléments réalistes, ce qui donne aux personnages davantage d'humanité, moins d'héroïsme que ce n'est le cas pour les protagonistes wagnériens. Une des marques les plus évidentes de cet éloignement formel du mythe tristanien est constituée par l'omniprésence du thème de l'enfant. Or, Tristan et Isolde ne sauraient être pensés avec des enfants. "Dépasser Tristan », cela peut vouloir dire ensuite remettre en question le sublime, l'emphase et le lyrisme tristaniens. Les figures attendues du tristanisme théâtral, en particulier le fameux « duo d'amour ", sont, avec humour ou sens pudique de l'euphémisme et du non-dit, différées ou transformées. Le refus du ton tristanien revient de façon plus globale à un refus du nihilisme wagnérien. Le romantisme tristanien est un épisode de l'histoire esthétique et philosophique de l'Europe sur lequel les plus critiques de ces pièces souhaiteraient tourner la page.

Tristan parodié : la hantise d'une fin de l'art conjurée?

Le Tristan de Wagner, de Fontane à Joyce et Brecht, en passant par Céard et T. Mann, a fait l'objet de multiples parodies. Pourquoi cela? Tout d'abord - ainsi s'en expliquent certains - parce que la parodie wagnérienne serait une nécessité, une affaire de salubrité culturelle. Wagner en effet stérilise l'artiste, énerve le spectateur, son œuvre le terrasse et l'écrase. La seule issue, devant tant de sublime, tant de sérieux, tant de prétention, dangereux pour l'état moral et physique de l'être et de la communauté, est d'en rire. C'est Nietzsche qui a le mieux mis en avant cette nécessité de démonter l'édifice wagnérien par la parodie. Transformer le prosaïque en langage wagnérien, exprimer le réel dans "la langue du maître", c'est l'enjoliver, lui donner une profondeur qui frise la vacuité et la grandiloquence. À l'inverse, transposer Wagner dans le réel bourgeois, c'est s'assurer, dans le rire et le divertissement, une guérison 
accélérée de l'empoisonnement wagnérien. Nietzsche offre plusieurs pistes possibles de parodies, dont certaines semblent avoir été prises au pied de la lettre par des écrivains tels que Mann, Wedekind, ou Céard (Terrains à vendre au bord de la mer, 1906), des parodies qui mettent en scène non pas des héros mythologiques, mais « le contenu réel de ces mythes ». Ernst Bloch demande quant à lui, dans Héritage de ce temps (Erbschaft dieser Zeit, 1935) que l'on fasse subir à son œuvre l'épreuve carnavalesque. Le seul moyen, dit Bloch, de retrouver une certaine authenticité de l'œuvre wagnérienne « pardelà la pacotille onirique » du dix-neuvième siècle bourgeois, c'est de les porter sur les tréteaux de la foire, de lui faire subir l'épreuve du roman de colportage. Enfin, dans Le Docteur Faustus de T. Mann, Leverkühn avoue ses réticences à se consacrer pleinement à la musique. La plus importante est qu'il dit ne plus croire à l'œuvre d'art, à cette donnée obligatoirement mystificatrice que manie si bien Wagner. La parodie tristanienne apparaît alors non plus comme une émancipation lucide, mais comme un destin irrémédiable de toute littérature placée dans le sillage wagnérien, une fatalité propre à l'œuvre d'art moderne en général. Si la littérature wagnérienne parodie Wagner, c'est qu'elle en ressent le besoin tout autant qu'elle n'en a pas le choix. S'y exprime la nostalgie des utopies volontairement perdues.

Cela, on le remarque particulièrement, chez D'Annunzio, Mendès (Le chercheur de tares, 1898), Hesse, Céard, Lawrence, Schnitzler (Vienne au Crépuscule [der Weg ins freie, 1908]) et bien d'autres, dans le cas particulier des figures de dilettantes wagnériens. Ce dont il retourne ici est l'adéquation entre l'appel à la création ressenti au contact du Tristan de Wagner, et son impossibilité à rendre effective cette réalisation dans le monde dans lequel évolue le wagnérien. Le dilettantisme tragique, ce décadentisme typique, est un des traits essentiels du tristanisme en tant que maladie de civilisation. Plusieurs invariants de la figure du dilettante wagnérien permettent de se rendre compte de cet aspect, invariants qui tous gravitent autour d'un refus tristanien du principe de réalité. Dans toutes ces œuvres en effet on remarque l'établissement d'un hiatus, hiatus entre un amour à vivre et, concomitamment à ne pas vivre, entre une œuvre à faire mais infaisable, voire entre une politique, une action à mener mais qui ne sont pas menées. Le dilettante wagnérien singe le génie ; il " papillonne », hésite entre différentes formes d'existence et il n'arrive pas à se fixer; il pratique le dilettantisme comme un épicurisme de l'esprit pour tromper l'ennui métaphysique; enfin il ne se perçoit comme créateur qu'au contact de l'art - et non à partir de la relation que le Moi entretient avec le monde -, autrement dit : la sensibilité artistique constitue pour lui un leurre dans l'appréciation qu'il peut faire de ses propres facultés.

À travers la figure du dilettante wagnérien, l'écrivain exorcise ses propres angoisses éprouvées au contact de l'œuvre wagnérienne, une peur d'être lui-même condamné par l'art wagnérien à jouer le rôle d'un dilettante. Cette peur, déclarée plus ou moins implicitement par de nombreux écrivains, prend deux formes. La première est celle qui présuppose que le compositeur et son œuvre ont signé une forme d'achèvement de l'art, achèvement par lequel la perfection signerait en même temps que la finalité suprême de l'art, rien d'autre que sa fin historique. Cette fin de l'art condamnant alors tout artiste à n'être qu'un épigone, un dilettante. La seconde peur est plus vaste et met en lumière une sorte d'impasse esthétique dont Wagner serait le symbole: Wagner aurait été le dernier à pratiquer l'utopie d'un lien esthético-politique entre l'artiste et son public; à faire croire que l'Idée pourrait en ce monde trouver son incarnation 
plastique. Après lui, non seulement la réalisation pratique de l'Un et du Total, mais aussi l'utopie même de cette réalisation auraient définitivement battu de l'aile.

Bilan : métamorphoses du mythe sous le patronage de Wagner

Romans, pièces de théâtre, opéras, textes poétiques divers, essais : le Tristan de Wagner a donné lieu au spectre des formes littéraires le plus large possible. On peut brièvement en donner la typo-chronologie suivante.

La première série de textes relève d'un tristanisme décadent. C'est un tristanisme de l'excès, qu'il s'agisse d'excès thématiques ou d'excès formels : pessimisme mortifère, surenchérissement kitsch de la longueur et les moyens. Cet excès, marque d'une incomplétude ou d'une imperfection fondamentale, replonge l'œuvre à prétention mythique dans l'histoire, le particularisme et la contingence. Il devient difficile de hisser la matière, qui perd son primordial caractère d'évidence, au niveau du mythe. L'exhibition renforcée des données du mythe tristanien, leur soulignement esthétique crispé, l'ajout de considérations sur la stérilité artistique qui viennent redoubler le thème wagnérien de l'impuissance et de la souffrance métaphysiques des personnages, finit par confiner au pur esthétisme, un esthétisme décadent qui les replace au centre même du processus historique qu'elles prétendaient éradiquer. L'excès, signe d'un vide nouveau autour duquel gravite une œuvre devenue bancale, lui fait encourir, même dans les cas les plus réussis, le risque du kitsch, de la complaisance esthétisante, et de la tautologie. Le drame métaphysique des héros wagnériens se dégrade en l'image d'individus confrontés dans une cyclicité sans fin à la crispation répétitive de leurs propres désirs. De fable, de pure fiction sécrétée par la société à la façon d'une nécessité jusqu'à la dimension du culte, le tristanisme, quittant par bovarysme l'espace de la fiction, devient une banale mais terrible maladie de civilisation. Elle ne pouvait qu'être décadente dans la mesure même où l'être réel, dont la maladie consiste à ne plus pouvoir séparer réel esthétisé, enjolivé, et fiction sublime et sacrée, ne peut faire autrement que se ressentir comme démuni face au héros tristanien. Du sentiment de décadence, l'individu, se condamnant lui-même à l'impuissance et au dégoût de soi, devient réellement décadent : un jouisseur, un esthète, un impuissant, mais aussi un malade authentique, aussi bien du point de vue physiologique que psychanalytique. Le tristanisme, est non seulement un mal de civilisation que l'on peut désormais esthétiquement dater, une sorte d'impasse, mais aussi la condition même de l'artiste moderne, hanté par le spectre de la stérilité et du dilettantisme.

31 La deuxième série de textes tristaniens témoigne d'une prise de conscience par rapport à ce tristanisme comme maladie de civilisation et intègre cette prise de conscience dans ses textes, qui subissent un certain détachement critique, ironique ou parodique : le tristanisme sérieux se poursuit, certes, mais de façon critique, réécrite, alternative. Ainsi, on refuse les présupposés hyperboliquement pessimistes de l'œuvre wagnérienne, son fond métaphysique, germanique, ses traits schopenhaueriens ou orientalisants. On lui donne un autre éclairage métaphysique. La désolidarisation des matières tristaniennes, de subies, commencent à être en partie choisies: on accepte que se séparent la mystique d'Eros et sa manifestation sensuelle unilatérale; le corps souffrant ne l'est plus symboliquement mais réellement ; les personnages perdent leur statut mythique initial pour ne plus y accéder qu'à mi-parcours des œuvres en question. Le retour de l'histoire venant affleurer dans un mythe qui se disloque commence à être accepté et intégré à l'œuvre. On se moque des entichements à répétition dont sont gratifiés les chanteurs wagnériens, aussi sublimes à la scène que 
triviaux à la ville. Partition évoquée dans le chuchotement, touchée avec précaution, commentaires devenus clichéiques sur la mort d'amour, la prose oxymorique de l'œuvre, son hymne nocturne, son chromatisme brûlant: on se met à se moquer des excès de ritualisation et de sacralisation dont jouit en particulier cette œuvre wagnérienne. Le décadentisme tristanien est ensuite reversé sur l'œuvre wagnérienne elle-même, dont on exhibe des traits que l'on commence à considérer comme étant des marques de décadence : révéler le fond vénitien latent de Tristan devient ainsi un moyen pour dire son décadentisme consubstantiel. On demande enfin des Tristan moins univocément sublimes, métaphysiques que ne l'est l'œuvre wagnérienne. Même si les écrivains conservent une relation ambiguë avec ce deuil du génie, de l'œuvre totale, et des présupposés métaphysiques qui leur sont liés, cette donnée laisse moins planer son ombre angoissée dans la création littéraire tristanienne. Le drame de l'artiste tristanien, condamné au dilettantisme ou à l'imitation, est exprimé de façon beaucoup moins tragique. La parodie, la démystification érotisante sont pensées comme des moyens de rendre compte de l'impasse tristanienne. Elles n'empêchent pas les écrivains de sentir, de façon lucide, mi-amusée, mi-résignée, que la parodie n'est qu'une sorte de pis-aller par rapport à l'hypotexte qu'elle prend à charge, qu'une échappée ambiguë par rapport au spectre angoissant de la non-création, de l'art épigonal. Mais l'art en mineur d'une œuvre qui se présente outrageusement elle-même comme majeure finit par représenter un âge de raison, une maturité de la littérature, son accession à la modernité, placée sous le signe de la référencialité ludique.

La dernière série de textes tristaniens se caractérise par un stade avancé, terminal, de réécriture du mythe. Tristan, chez Joyce, Leiris, Gracq (La Presqu'île, 1970), Robbe-Grillet (Le miroir qui revient, 1984), Balestrini (Tristano, 1964) ou Treichel (Tristanakkord, 2001), devient un signifiant censé fonctionner par et pour lui-même, si riche d'histoire qu'il n'est plus besoin de le vivifier quand on l'évoque. C'est un stimulus pour l'imagination collective, un effet de captatio sur le lecteur. Il en résulte que le signifié lui-même finit, dans cette autosuffisance du signifiant, par se vider de tout contenu. La sacralité, le mysticisme du tristanisme ne sont plus évoqués que pour en souligner l'absence en ce monde, absence considérée comme bénéfique ou au contraire comme catastrophique, et mise en parallèle avec l'affadissement du sacré en général, du christianisme en particulier, sphère avec laquelle il avait un rapport aussi privilégié que contradictoire. Le tristanisme est une donnée en voie de sédimentation, en voie de devenir un fragment culturel parmi d'autres, se perdant dans la conscience collective comme les ruines d'un ancien temple dans la sylve des éléments de culture plus actifs. L'omniprésence d'un tristanisme dégradé dans l'art de masse après s'être fourvoyé dans le culte bourgeois de la beauté montrerait la disparition du véritable foyer tristanien, proche du foyer divin. Eros s'évapore pour ne plus concerner que la littérature elle-même. Le désir tristanien devient alors celui du lecteur cultivé : la référence mythologique voilée mais décodée, le contrat mythologique rempli ou au contraire différé, modifié, sont des moyens de créer une sorte d'érotique de la lecture. 


\section{NOTES}

1.D. de Rougemont, L'amour et l'Occident, Paris, Plon, 1972 [1938 et 1956], p. 253.

2.Lettre à Mathilde Wesendonck du 10 avril 1859.

3.Prologue à Tristan et Isolde, L'Avant-Scène Opéra, 34-35, p. 36.

4.Sur la question des sources médiévales du Tristan de Wagner, voir D. Buschinger, Le Moyen Âge de Richard Wagner, Université de Picardie - Jules Verne, Amiens, 2003.

5.Lettre à Liszt du 16 décembre 1854.

6.Sur Tristan et Schopenhauer, voir E. Sans, Richard Wagner et la pensée schopenhauerienne, Paris, Klincksieck, 1969.

7.Sur ce point, cf. M. Petitjean, «L'extase tristanienne, une initiation métaphysique », Paris, L'Avant-Scène Opéra, 34-35, pp. 154-163.

8.F. Nietzsche, La Naissance de la tragédie, Paris, Gallimard, pp. 124-129.

9.Cf. T. Mann, Wagner et notre temps, trad. F. Bertaud, Paris, Le Livre de poche, Pluriel, 1978.

10.Les principaux textes que Claudel consacre à Wagner sont Richard Wagner, Rêverie d'un poëte français (1927) et Le poison wagnérien (1938).

11.Lettre de décembre 1907 à Jacques Rivière.

12.P. Claudel, Richard Wagner, Rêverie d'un poëte français, Paris, les Belles Lettres, 1970,

p. 63.

13.Propos tirés de la préface à la réédition de 1948 de Partage de midi.

14.P. Claudel, Mes idées sur le théâtre, Paris, Gallimard, 1966, p. 59.

15.Voir la plupart des essais contenus dans Création littéraire et connaissance (Dichten und Erkennen, 1955).

16.Cf. les Notes sur L'Opéra de quat'sous $(1929,1931$ et 1937) et Notes sur l'opéra Grandeur et décadence de la ville de Mahagonny (1930 et 1937).

17.H. Marcuse, Eros et Civilisation [Eros and Civilization, 1955], Paris, Minuit, 1963, p. 109.

18.Dans son édition de Chair mystique, Séguier, Bibliothèque décadente, 1995, Jean de Palacio recense quelques vingt-cinq Tristan romanesques et poétiques entre 1865 et 1923.

19.Ainsi des passages sur Tristan dans La Prisonnière.

20.De Jean d'Agrève de M. de Vogué (1897) à Aurélien d'Aragon (1944, chapitre 45), les romans du couple amoureux placés explicitement sous le patronage du Tristan de Wagner sont fort nombreux.

21.Cf. A. Corbellari, Joseph Bédier écrivain et philologue, Genève, Droz, 1997.

22.Dans Love, Honour and Artifice (Kümmerle Verlag, Göppingen, 1989), ouvrage consacré aux adaptations théâtrales du mythe de Tristan, E. Poletti recense, dans les pays de langue allemande, plus d'une vingtaine de pièces entre 1853 et 1919. 


\section{AUTEUR}

TIMOTHÉE PICARD

Université Strasbourg-II 